\title{
EXTREMADURA, VERDE, BLANCA, NEGRA. AVANCES EN ALTAS CAPACIDADES
}

\author{
Carmen Gómez Labrador \\ Universidad de Extremadura: Departamento de Psicología y Antropología \\ carmengomezlab@gmail.com \\ Margarita Gozalo Delgado \\ Universidad de Extremadura: Departamento de Psicología y Antropología \\ Benito León del Barco \\ Universidad de Extremadura: Departamento de Psicología y Antropología \\ Santiago Mendo Lázaro \\ Universidad de Extremadura: Departamento de Psicología y Antropología
}

Fecha de Recepción: 4 Enero 2019

Fecha de Admisión: 30 Abril 2019

\section{RESUMEN}

La atención al alumnado de AACC presenta muchas diferencias en función de las políticas educativas de las distintas Comunidades Autónomas. Existe una amplia variabilidad tanto en la consideración teórica de las altas capacidades intelectuales, como en la atención que recibe este alumnado en el marco autonómico, siendo los programas de detección /enriquecimiento desarrollados, la formación que reciben los docentes (cursos, postgrados, etc) y las actitudes hacia la consideración de la alta capacidad, recursos que suponen una ventaja 0 una desventaja a la hora de afrontar el reto que supone la identificación y atención a las necesidades educativas de estos alumnos en la escuela inclusiva. Como consecuencia de estas diferencias, el número de alumnos identificados varían sustancialmente entre aquellas comunidades que empezaron en décadas pasadas a invertir recursos en la atención de este alumnado y otras que tienen una menor trayectoria en su inclusión educativa.

La normativa vigente en Extremadura plantea a las familias y a los profesionales de la educación dificultades para la atención de las necesidades educativas de este alumnado. Entre ellas podemos encontrar la formación, creencias y actitudes del profesorado y su implicación en la identificación, evaluación y respuesta educativa a estos alumnos. Sin embargo, en los últimos años se observan cambios en este panorama, con distintas acciones a favor de este colectivo, desarrolladas tanto desde la administración educativa como desde movimientos asociativos y entidades privadas.

En este trabajo nos proponemos explorar la situación actual de la atención a este alumnado en Extremadura, respecto al de otras comunidades autónomas, exponer y valorar las propuestas que 
se han desarrollado más recientemente y se han traducido en programas específicos desarrollados por iniciativa de los propios centros educativos, con actuaciones extracurriculares a nivel público y privado que intentan responder a las necesidades específicas de apoyo educativo que presenta este alumnado.

Palabras clave: altas capacidades; identificación; actitudes; inclusión educativa; talento; atención a diversidad

\section{ABSTRACT}

Extremadura, green, white, black. Advances in high capacities. The attention to the students of AACC presents many differences according to the educational policies of the different Autonomous Communities. There is a wide variability both in the theoretical consideration of the high intellectual capacities, and in the attention that these students receive in the autonomic framework, being the detection / enrichment programs developed, the training that the teachers receive (courses, postgraduates, etc.) and attitudes towards the consideration of high capacity, resources that suppose an advantage or a disadvantage when facing the challenge that involves the identification and attention to the educational needs of these students in the inclusive school. As a consequence of these differences, the number of students identified varies substantially between those communities that began in past decades to invest resources in the care of these students and others that have less experience in their educational inclusion.

The current regulations in Extremadura pose difficulties for families and education professionals to meet the educational needs of these students. Among them we can find the training, beliefs and attitudes of teachers and their involvement in the identification, evaluation and educational response to these students. However, in recent years there have been changes in this panorama, with different actions in favor of this group, developed both from the educational administration and from associations and private entities.

In this paper we propose to explore the current situation of care for these students in Extremadura, compared to other autonomous communities, expose and assess the proposals that have been developed more recently and have been translated into specific programs developed at the initiative of the centers themselves educational, with extracurricular performances at public and private level that try to respond to the specific educational support needs presented by these students.

Keywords: high abilities; ID; attitudes educational inclusión; talent; attention to diversity

\section{INTRODUCCIÓN}

Uno de los problemas más evidentes de nuestro sistema educativo, es su falta de flexibilidad y atención individualizada a la diversidad del alumnado. El profesorado mantiene el principio de igualdad en la educación dejando a un lado el de la atención a la diversidad y nuestra escuela clasifica a los alumnos en función de la edad y no de su capacidad, proponiendo un currículo cerrado, con el mismo nivel de dificultad para todos al margen de sus fortalezas y debilidades. Aquellos alumnos con niveles de desarrollo y potencial cognitivo superiores a los esperados según su edad, muchas veces sufren las consecuencias de este modelo, y sus capacidades pasan inadvertidas o son ignoradas por muchos docentes.

La situación de este alumnado en Extremadura, aún dista mucho de la que tienen en otras Comunidades, siendo difícil su identificación temprana, su consideración como alumnos de altas capacidades y su respuesta educativa. Encontramos todavía docentes que desconocen qué significa que un alumno tenga altas capacidades intelectuales y cómo atender a sus necesidades educativas. Profesores que suponen que un alumno "brillante" lo es por la cantidad de estimulación que 
recibe y que estos alumnos tienen que ser excelentes en su rendimiento escolar, que nunca fracasan. Algunos sienten cuestionada su profesionalidad después de una larga trayectoria educativa sin haber detectado a ningún alumno con alta capacidad intelectual en sus aulas. Otros reconocen que no se sienten formados en esta temática y muestran prejuicios ante el reto que supone para ellos abordar la respuesta educativa de estos alumnos.

\section{ALTA CAPACIDAD, DIFICIL TÉRMINO A ACOTAR}

La alta capacidad intelectual, ha sido un término difícilmente definible, desde el entramado de teorías explicativas, diagnósticas, de evaluación o de intervención existentes en torno a él. Hoy día, conviven distintos modelos explicativos de este constructo, con distinta terminología que dificulta la comprensión de qué significa que un alumno tenga altas capacidades.

Haciendo un recorrido por las múltiples definiciones aportadas desde las distintas teorías, conceptualizamos la alta capacidad intelectual desde la inteligencia entendida como un factor generalizado, al modelo distintivo de superdotación y talentos, lo mismo ocurre con el conglomerado de términos con los que definimos a estos alumnos, desde el mal traducido e interpretado concepto de superdotado hasta el más idóneo y actualmente recomendado término de alta capacidad.

Esta ambigüedad sustenta la necesidad de matizar conceptos como Alta capacidad, atribuido a sujetos que presentan una amplia gama de capacidades y aprenden con facilidad cualquier área 0 materia. Miguel López y Moya Gutiérrez (2006) afirman que las diferencias son fundamentalmente cualitativas, esto es, presentan un funcionamiento diferente a la hora de enfrentarse y resolver una tarea. Según Jiménez Fernández (2014) los alumnos con altas capacidades, son aquellos que aprenden a mayor ritmo, con mayor profundidad y mayor amplitud que sus iguales, sobre todo si trabajan en temas que atraen su interés y si encuentran en padres y profesores el apoyo y la guía adecuados. Pfeiffer (2013) se refiere a tres alternativas a tener en cuenta para la visualización, la identificación, la agrupación y la educación de los estudiantes de capacidad poco común o alta, según lo que denomina el Modelo Tripartito, desde el que se considera esta alta capacidad a través de la lente de la alta inteligencia, del alto rendimiento y del potencial para rendir de modo excelente.

Las palabras "altas capacidades intelectuales" designan como término genérico a aquellos alumnos que presentan potencialmente alta capacidad en una, algunas o en la mayoría de las áreas de la inteligencia entendida en sentido amplio, pudiendo demostrar o no conductas propias de alumno excelente o muy por encima de la media en uno o varios ámbitos. No es solo tener un desarroIlo mayor de aptitudes intelectuales. Es una distinta configuración cerebral. Es algo biológico que va más allá de un $\mathrm{Cl}$ elevado. Las más recientes concepciones de la alta capacidad se refieren a ella como un potencial que se va desarrollando a lo largo de la vida (Bermejo, Salazar, Zapatera y Ferrando (2016), p.129) y a la vez como un comportamiento inteligente y aprendido. Para Elices, Palazuelo y Del Caño (2013) la diferenciación entre superdotación y talento (Gagné, 1995) es una de las propuestas más productivas y operativas, ya que aporta pautas claras para una mejor identificación de estos alumnos. Además, permite delimitar mejor cuáles pueden ser las necesidades educativas de cada uno de ellos, con objeto de implementar las medidas de atención oportunas.

\section{SITUACIÓN LEGISLATIVA ACTUAL DE ESTOS ALUMNOS}

Son muchas las variables que la respuesta educativa debe aunar no sólo desde la identificación sino también desde el punto de vista legislativo.

Desde el Dictamen del Comité Económico y Social Europeo (Dict.CEES, de 13 de enero de 2013) "Liberar el potencial de los niños y los jóvenes con gran capacidad intelectual dentro de la Unión Europea", se hace una recomendación a todos los estados miembros para que apoyen la realización de estudios e investigaciones adicionales y adopten medidas adecuadas que favorezcan la atención 
a la diversidad de todas las personas en general, incluyendo programas capaces de movilizar el potencial de los niños y los jóvenes con alta capacidad.. Poniendo de manifiesto la necesidad de mejorar las prácticas y actividades educativas destinadas a este tipo de alumnos.

En este contexto, el Programa Europa 2020: Una estrategia para un crecimiento inteligente, sostenible e integrador, aprobado por la Comisión Europea en 2010, incluye como una de sus tres prioridades básicas: "la búsqueda del crecimiento inteligente mediante el desarrollo de una economía basada en el conocimiento y la innovación". La educación de todos los ciudadanos se convierte en un recurso clave para garantizar el futuro de la Unión Europea, y esto incluye la mejora en la detección y atención educativa de las personas con altas capacidades.

En nuestro país nos encontramos con un marco conceptual confuso, tanto en el empleo de los términos utilizados referente al alumnado con alta capacidad, como en lo relativo a su identificación y medidas de atención educativa.

La actual legislación educativa (LOMCE 8/2013, de 9 de diciembre), no define el concepto de "altas capacidades intelectuales" ni aclara qué alumnos lo conformarían. Por tanto, la situación en España muestra diferentes niveles de desarrollo legislativo entre las Comunidades, respecto a la atención educativa que reciben, así como en los términos que se emplean para referirse a ello y en ocasiones, su definición conceptual, no se ajusta a los avances teóricos y científicos en la materia. De igual forma, introduce el concepto de altas capacidades, como término más general que el de superdotación y reclama la atención también sobre los talentosos, los niños/as precoces y sobre todo aquel alumnado que puede manifestar conductas propias del individuo bien dotado. Apoya más el carácter de potencialidad frente a la exigencia de rendimiento que recogían anteriores disposiciones legales. Sin embargo, la disparidad en nuestra legislación educativa sobre los criterios de identificación del alumnado con alta capacidad, dificulta la labor de detección, las actitudes de los docentes hacia ellos y la respuesta educativa que se propone.

Los Informes Anuales del Estado del Sistema Educativo, publicados anualmente por el Ministerio de Educación y Formación Profesional, insisten en la escasez de alumnos identificados en nuestro país, siendo dispares los datos de alumnos con altas capacidades si comparamos unas regiones con otras.

Según datos oficiales de la Consejería de Educación y Empleo de Extremadura al finalizar 2017, están identificados 306 alumnos (0.16\% de los alumnos escolarizados). Por debajo de la media nacional que se sitúa en el $0.29 \%$ del total de alumnos. Superan este porcentaje regiones como Murcia (con un $1.23 \%)$, Canarias (0.56\%), Andalucía (0.60\%), Asturias $(0.50 \%)$, Galicia $(0.36 \%)$, Baleares $(0.35 \%)$ y se encontrarían por debajo de este porcentaje comunidades como Navarra $(0,26 \%)$, Castilla y León $(0.17 \%)$, Cantabria $(0.14 \%)$, Madrid $(0.17 \%)$ y Extremadura, siendo regiones como País Vasco (con $0.10 \%$ ), Comunitat Valenciana (0.12\%), Aragón y Castilla-La Mancha (con $0.06 \%$ y 0.09 respectivamente) y Cataluña (con $0.03 \%$ ) las que menos alumnos con AACC identifican en sus aulas.

\section{Situación legislativa en Extremadura}

En el año 2002, bajo la normativa de la Ley Orgánica de Ordenación General del Sistema Educativo (LOGSE 1/1990, de 3 de Octubre), se promulga en Extremadura la Orden de 31 de julio de 2002, por la que se regula el procedimiento para orientar la respuesta educativa al alumnado con talentos específicos, alta capacidad o sobredotación intelectual. En el mismo marco normativo de la LOGSE, dos años después se publica la Orden de 27 de febrero de 2004, por la que se regula el procedimiento para orientar la respuesta educativa para los alumnos superdotados intelectualmente. Esta Orden sigue vigente en la actualidad, distando mucho de las nuevas concepciones teóricas sobre la alta capacidad. 
En el año 2011, dentro del marco normativo de la Ley Orgánica, de Educación (LOE 2/2006, de 3 de mayo), se publica la Ley de Educación de Extremadura (LEEX 4/2011, de 7 de marzo). En su Art.27. expone que la Administración Educativa adoptará medidas para la detección temprana, la valoración de necesidades y la atención educativa del Alumnado con Altas Capacidades Intelectuales.

En años posteriores, dentro del marco educativo actual de la Ley Orgánica de Mejora de la Calidad Educativa (LOMCE 8/2013 de 9 de diciembre) entra en vigor el Decreto por el que se establece el currículo de Educación Primaria para la Comunidad Autónoma de Extremadura (D 103/2014, de 10 de junio) en su Capítulo IV, Artículo 13, punto 5, recoge que "Las acciones preventivas y la detección temprana tendrán carácter prioritario en los centros educativos, con la implicación y la participación de toda la comunidad educativa y, en su caso, de otras administraciones 0 entidades sin ánimo de lucro, que deberán firmar acuerdos de colaboración con la Consejería".

El Decreto por el que se establecen la ordenación y el currículo de la Educación Secundaria Obligatoria y del Bachillerato para la Comunidad Autónoma de Extremadura (D 98/2016, de 5 de julio) en su capítulo II. Atención a la diversidad del alumnado. Art. 16.Alumnado con altas capacidades intelectuales expone que "La Consejería competente en materia de educación adoptará las medidas necesarias para identificar al alumnado con altas capacidades intelectuales y valorará de forma temprana sus necesidades".

En la necesidad de crear una normativa marco que facilitara a la comunidad educativa extremeña las pautas para el conocimiento y atención educativa a la diversidad, surge el Decreto por el que se regula la respuesta educativa a la diversidad del alumnado en la Comunidad Autónoma de Extremadura (Decreto 228/2014, de 14 de octubre). Respecto a los alumnos con altas capacidades, en el capítulo 1. Disposiciones Generales, en sus Principios de Actuación, art 3.5, expone que "Las acciones preventivas y la detección temprana tendrán carácter prioritario, debiendo asegurar la implicación y la participación de toda la comunidad educativa y, en su caso, de otras administraciones 0 entidades sin ánimo de lucro". Recoge una definición un tanto controvertida sobre qué es un alumno con altas capacidades, claramente desde los modelos orientados al rendimiento. Así, el art.17.1. Alumnado con Altas Capacidades Intelectuales, establece unos criterios con los que "identificar" a este alumnado en Extremadura. Expone dicho artículo que "se considera alumnado con necesidades específicas de apoyo educativo por altas capacidades intelectuales aquel cuya evaluación psicopedagógica determine que dispone de una capacidad intelectual superior a la media con evidencia de una elevada productividad, un alto nivel de creatividad y un alto grado de dedicación a las tareas".

La realidad muestra que Extremadura es una de las comunidades de España en la que menos alumnos con altas capacidades aparecen identificados. El siguiente gráfico muestra el alumnado identificado con altas capacidades en España por sexo, titularidad de centro y tipo de financiación de las enseñanzas en el curso 2016/2017. 
Tabla 1.

Número de Alumnos de Altas Capacidades identificados en España en función del Nivel Educativo y la Titularidad del Centro.

NIVEL EDUCATIVO TITULARIDAD DEL CENTRO

$\begin{array}{ccc}\text { Públicos Privados } & \begin{array}{c}\text { Privados no } \\ \text { concertados }\end{array} & \begin{array}{c}\text { Todos los } \\ \text { centros }\end{array}\end{array}$

\begin{tabular}{|c|c|c|c|c|}
\hline $\begin{array}{l}\text { Segundo ciclo de } \\
\text { Educación Infantil }\end{array}$ & 134 & 62 & 8 & 204 \\
\hline Educación Primaria & 10175 & 3475 & 284 & 13934 \\
\hline $\begin{array}{l}\text { Educación Secundaria } \\
\text { Obligatoria }\end{array}$ & 6647 & 2649 & 240 & 9536 \\
\hline Bachillerato & 2875 & 214 & 232 & 3321 \\
\hline $\begin{array}{l}\text { Formación Profesional } \\
\text { Básica }\end{array}$ & 10 & 0 & 0 & 10 \\
\hline $\begin{array}{l}\text { Ciclos Formativos de } \\
\text { Grado Medio }\end{array}$ & 58 & 6 & 0 & 64 \\
\hline $\begin{array}{l}\text { Ciclos Formativos de } \\
\text { Grado Superior }\end{array}$ & 60 & 4 & 0 & 64 \\
\hline Total & 19959 & 6410 & 764 & 27133 \\
\hline
\end{tabular}

La falta de formación docente que facilite esa identificación, sustenta esta estadística. Esta Formación Permanente del Profesorado en Extremadura se hace principalmente en los CPR (Centro de Profesores y Recursos) siendo los cursos impartidos sobre la temática de las altas capacidades más solicitados que en años anteriores, si bien aún muy escasos en general. Así, el año 2015 se imparten 2 cursos (en CPR de Trujillo y Cáceres), en 2016, 3 cursos (2 en CPR de Almendralejo y 1 en Cáceres), en 2017 , 5 cursos (1 en Badajoz, 1 en Cáceres, 1 en Plasencia y 2 en CPR de Coria), en 2018 se imparten 4 cursos ( 1 en Caminomorisco, 1 en Almendralejo, 1 en Coria, 1 en Cáceres). En el año 2019, se ha impartido un curso en Almendralejo y una charla en CPR de Cáceres.

Además, una Jornada desde la Administración para los orientadores y formación puntual en aquellos centros en los que se ha desarrollado el programa Proyect@ desde el año 2017. Igualmente desde la Universidad de Extremadura se imparten desde el año 2018 cursos de perfeccionamiento dirigidos a los futuros docentes.

\section{ACTUACIONES EDUCATIVAS MÁS RECIENTES CON ESTE ALUMNADO EN EXTREMADURA.}

En febrero de 2017, en el marco legislativo autonómico referenciado anteriormente, surge el programa experimental para el desarrollo de capacidades Proyect@, en Extremadura. Si bien en el curso, académico 2017-18, se ofertó sólo para educación secundaria, en el curso 2018-19 se inclu- 
yó también Educación Primaria. El programa no solo se dirige a alumnado que presenta necesidades específicas de apoyo educativo derivadas de altas capacidades intelectuales (ya que la identificación de estos alumnos está muy por debajo de lo esperado en nuestra Comunidad) sino también a aquellos con habilidades especiales para algún tipo de área en concreto, alumnado con disposición positiva a la dedicación de parte del tiempo libre al desarrollo de proyectos de investigación, desarrollo e innovación en el ámbito escolar, alumnos con alto grado de dedicación y motivación para la realización de tareas para el aprendizaje y aquellos con nivel de rendimiento y aprovechamiento educativo elevado.

Actualmente, en el primer semestre de 2019, son 18 colegios de educación primaria y 53 institutos de educación secundaria, los que desarrollan este proyecto en toda Extremadura, de forma voluntaria y en horario extraescolar.

Algunas de estas actuaciones, enfocadas como experiencias de éxito e innovación educativa en Ios centros, fueron expuestas en las I Jornadas de Altas Capacidades en Extremadura, celebradas en junio de 2018, donde desde la Consejería de Educación y Empleo se expuso la necesidad de trabajar a nivel institucional en la identificación y en el reconocimiento de los distintos perfiles de alumnos de AACC. Fueron un éxito de asistencia de toda la comunidad educativa, con participación de orientadores, docentes y familias.

En algunos centros educativos están apostando por realizar programas de identificación de este alumnado y programas de enriquecimiento con los que dotar de identidad a la institución que los lleva a cabo, pasando de ser un hecho puntual y aislado en pocos centros a ser una práctica ofertada en este último curso para atraer alumnado con estas características a determinados centros educativos.

Como iniciativa desde el ámbito privado, surge el Programa de Enriquecimiento TalentiaCC en febrero de 2018, en el que han participado más de 50 niños y adolescentes de AACC de toda Extremadura y sus familias. Se ha llevado a cabo a través de talleres diseñados específicamente para este tipo de alumnado, sobre temas de interés, incluyendo sesiones de formación para los padres. El objetivo principal del programa ha sido desarrollar los posibles intereses de estos alumnos desde una perspectiva extracurricular, incluyendo actividades de diferentes temáticas que satisfagan sus intereses y las necesidades de tipo social y emocional que estos niños presentan, de forma complementaria a su experiencia en los Centros Educativos.

\section{CONCLUSIÓN}

Al igual que en otras comunidades, son muchos los inconvenientes que en Extremadura se registran a la hora de atender educativamente a este alumnado, desde la primera detección por la familia a las dificultades en cuanto a la identificación de sus necesidades por docentes inexpertos en el ámbito de las altas capacidades, así como en la adecuada respuesta educativa. La inexistencia de un marco teórico fundamentado en nuestra legislación, fomenta la disparidad de criterios con los que son considerados educativamente.

Seguimos encontrándonos en las aulas alumnos que se sienten incomprendidos, rechazados, abocados a fracasar en etapas posteriores por su desmotivación y su falta de estímulo hacia los aprendizajes estructurados en el currículo. La formación del profesorado es un paso fundamental para la detección y la respuesta educativa al alumnado con altas capacidades intelectuales.

Autores como Tourón (2015) abogan por un cambio real en el sistema educativo, atendiendo a la individualidad de cada persona con altas capacidades, innovando en metodologías y nuevos modelos de aprendizaje que consideren al alumno por su capacidad no por su edad.

En conclusión, los distintos criterios para identificar a estos alumnos, la coordinación con las familias, la falta de formación docente que facilite esa identificación así como la intervención en el 
cambio de actitudes favorables hacia este colectivo, resultan cuestiones especialmente relevantes para conseguir normalizar la atención educativa que demandan los alumnos con altas capacidades intelectuales en los centros de Extremadura. Se va avanzando en la consideración de este alumnado en nuestra Comunidad, desterrando tópicos relacionados con el $\mathrm{Cl}$ y el rendimiento académico en estos alumnos, haciendo visible la necesidad de mejorar la formación del profesorado para identificar y atender educativamente a este colectivo, siendo cada vez más los centros que llevan a cabo experiencias de innovación y buenas prácticas educativas que fomentan la motivación y el trabajo con estos alumnos y por tanto el desarrollo de su potencial.

\section{REFERENCIAS BIBLIOGRÁFICAS}

Bermejo, R., Salazar, M., Zapatera, A., y Ferrando, M. (2016). Cómo evaluar el talento matemático: un estudio piloto en estudiantes de Educación Secundaria. En I Congreso Internacional: nuevas perspectivas en el estudio de la Superdotación y el Talento (pp.131-135). Universidad de Murcia. Murcia. España.

Elices, J.A., Palazuelo, M., y Del Caño, M. (2013). Alumnos con altas capacidades intelectuales. Características, evaluación y respuesta educativa. Madrid. CEPE.

Gagné, F. (1995). From giftedness to talent: A developmental model and its impact on the language of the field. Roeper Review, 18, 103-111.

Gómez Labrador, C., Gozalo Delgado, M., Rubio Jiménez, J.C., Mendo Lázaro, S. (Julio de 2017). Intervención sobre las actitudes de los docentes de un centro educativo hacia el alumnado con altas capacidades. En Santolaya Ochando, F., (presidencia). III Congreso Internacional de Psicología. Colegio de Psicólogos. Oviedo, España.

Jiménez Fernández, C. (2014). El desarrollo del talento: educación y alta capacidad. Lección inaugural. Facultad de Educación. UNED. Madrid.

Martínez Torres, M. y Guirado, A. (2010) Altas Capacidades Intelectuales. Pautas de actuación, orientación, intervención y evaluación en el periodo escolar. Barcelona: Grao

Miguel López, A. y Moya Gutiérrez, A. (2006). Conceptos Generales del alumnado con altas capacidades. En Alumnos con altas capacidades y aprendizaje cooperativo. Un modelo de respuesta educativa. (pp.13-33). Fundación SM. Madrid.

Consejo Escolar del Estado (2015). Informe 2015 sobre el Sistema Educativo. Secretaría General Técnica. Recuperado de https://www.mecd.gob.es/educacion/mc/cee/publicaciones/informes-delsistema-educativo/informe-2015.html

Tourón, J., Santiago, R. (2015). El Modelo Flipped Learning y el desarrollo del talento en la escuela. Revista de Educación, 368, 196-231 España.

Pérez, L. y Domínguez, P. (2000). Superdotación y Adolescencia. Características y necesidades en la Comunidad de Madrid. Consejería de Educación. Comunidad de Madrid.

Pfeiffer, S.I. (2013) Serving the gifted: Evidence-based clinical and psychoeducational practice. New York: Routledge.

\section{REFERENCIAS LEGALES}

Unión Europea. Dictamen del Comité Económico y Social Europeo sobre el tema «Liberar el potencial de los niños y los jóvenes con gran capacidad intelectual dentro de la Unión Europea» (Dictamen de iniciativa) (2013/C 76/01). Diario Oficial de la Unión Europea, 486⿳⺈ sesión plenaria de los días 16 y 17 de enero de 2013.

España. Ley Orgánica 8/2013, de 9 de diciembre, para la mejora de la calidad educativa. Boletín Oficial del Estado, 12 de diciembre de 2013, núm. 295, pp. 97858- 97921. 
España. Ley 4/2011, de 7 de marzo, de Educación de Extremadura. Diario Oficial de Extremadura, 9 de marzo de 2011, núm.47, pp. 5952- 6035. Boletín Oficial del Estado, 23 de marzo de 2011, núm. 70 , pp. $30994-31060$.

España. Decreto 228/2014 de 14 de octubre, por el que se regula la respuesta educativa a la diversidad del alumnado en la Comunidad Autónoma de Extremadura. Diario Oficial de Extremadura, 21 de octubre de 2014, núm. 202, pp. 31554-31588.

España. Instrucción 2/2015 de la SGE por la que se concretan determinados aspectos sobre atención a la diversidad, según lo establecido en el Decreto 228/2014 de 14 de octubre, por el que se regula la respuesta educativa a la diversidad del alumnado de la comunidad autónoma de Extremadura. pp. 1-20.

España. Orden de 31 de julio de 2002, por la que se regula el procedimiento para orientar la respuesta educativa al alumnado con talentos específicos, alta capacidad o sobredotación intelectual. Diario Oficial de Extremadura, 31 de agosto de 2002, núm.101, pp.11115-11129.

España. Orden de 27 de Febrero de 2004, por la que se regula el procedimiento para orientar la respuesta educativa para los alumnos superdotados intelectualmente. Diario Oficial de Extremadura, 11 de marzo de 2004, núm. 29, pp. 2576-2595.

España. Decreto 103/2014, de 10 de junio, por el que se establece el currículo de Educación Primaria para la Comunidad Autónoma de Extremadura. Diario Oficial de Extremadura, 16 de junio de 2014, núm.114, pp. 18965-19283.

España. Decreto 98/2016, de 5 de julio, por el que se establecen la ordenación y el currículo de la Educación Secundaria Obligatoria y del Bachillerato para la Comunidad Autónoma de Extremadura. Diario Oficial de Extremadura, 6 de julio de 2016, núm.129, pp.17347-18550.

España. Real Decreto 1801/1999, de 26 de noviembre, sobre traspaso de funciones y servicios de la Administración del Estado a la Comunidad Autónoma de Extremadura en materia de enseñanza no universitaria. Boletín Oficial del Estado, 21 de diciembre de 1999, núm.304, pp. 4471044713.

Consejo Escolar de Extremadura.(2013). Informe sobre el Estado del Sistema Educativo en Extremadura. Recuperado de http://www.educarex.es/pub/cont/com/0002 /documentos/Informe_Consejo_Escolar_2012-13.pdf

Consejo Escolar del Estado. (2015).Informe del Estado del sistema educativo. Recuperado de http://www.mecd.gob.es/en/educacion/mc/cee/publicaciones/informes-del-sistemaeducativo/informe-2015.html

Consejo Escolar del Estado. (2018).Informe del Estado del sistema educativo. Recuperado de http://ntic.educacion.es/cee/i2018cee/ 
